\title{
EUV and Microwave Observation of a Filament
}

\author{
F. Chiuderi-Drago ${ }^{1}$
}

DASOP-Observatoire de Paris-Meudon, 92195 Meudon Cedex, France

K. Bocchialini, S. Lamartinie, J.-C. Vial

Institut d'Astrophysique Spatiale, Bât.121, Université Paris XI-CNRS, 91405 Orsay Cedex, France

T. Bastian

National Radio Astronomy Observatory, Soccoro, NM 87801, USA

C.E. Alissandrakis

Section of Astro-Geophysics, Department of Physics, Universiy of Ioannina, 45110 Ioannina, Greece

R. Harrison

Space Science Department, Rutherford Appleton Laboratory, Chilton, Didcot Oxfordshire OX11, OXX, UK.

J.-P. Delaboudinière, P. Lemaire

Institut d'Astrophysique Spatiale, Bât.121, Université Paris XI-CNRS, 91405 Orsay Cedex, France

\begin{abstract}
Simultaneous XUV and microwave $(\mu-w)$ observations of a solar filament, performed by several instruments onboard SOHO and by the Very Large Array (VLA), are analyzed. The filament appears as a dark structure, very similar in shape to the optical $H_{\alpha}$ filament, in all images taken in the transition region (TR) lines observed by CDS, in the $\mathrm{Ne}$ VIII lines observed by SUMER at $\lambda=770 \AA$ and $780 \AA$ and at all radio wavelengths with $1.7 \mathrm{~cm} \leq \lambda \leq 6 \mathrm{~cm}$. Contrary to that, in six TR lines observed by SUMER at $\lambda>1300 \AA$ no trace of the filament, either in absorption or in emission, is visible. Finally, at $\lambda=21 \mathrm{~cm}$, as well as in all images taken in coronal lines by CDS and EIT, a dark feature is present at the filament position, although with less defined contours than in the first-mentioned TR images. The constraints imposed by all these observations are discussed and interpreted.
\end{abstract}

\footnotetext{
${ }^{1}$ Current address: Dipartimento di Astronomia, University of Florence, Largo E.Fermi 5, 50125 Firenze, Italy.
} 


\section{Introduction}

A joint campaign to observe a filament on the Solar disk in the EUV and $\mu-w$ range of wavelengths has been proposed having in mind the following scientific motivation:

Previous observations of prominences in EUV lines (Schmahl and Orrall, 1986; Engvold, 1989), have supplied the Differential Emission Measure ( $D E M=$ $N^{2} d h / d T$ ) as a function of temperature in the Prominence-Corona Transition Region (PCTR). If this quantity is used to compute the radio optical depth $(\tau \propto D E M)$ and the corresponding brightness temperature, $T_{b}$, at $\mu-w$, the results strongly disagree with the observations with $T_{b}(o b s) \ll T_{b}($ calc $)$. It must be pointed out that the above EUV observations were all performed on prominences observed at the limb, while all radio observations refer to filaments on the disk (Chiuderi Drago 1990).

This strong discrepancy was interpreted as due to a real difference between the physical parameters of the PCTR at the prominence top (disk observations) and at its side (limb observations) because of the different orientation of the magnetic field with respect to the temperature gradient. If the angle $\theta$ between these two directions is taken into account in the heat conduction, a first integral of the energy equation shows that the DEM scales as $\cos \theta$ (Chiuderi and Chiuderi Drago 1990). Since, according to Leroy (1989), $\theta \simeq \pi / 2$ at the prominence top, while it is $30^{\circ} \leq \theta \leq 90^{\circ}$ on the side, the DEM is much lower at the prominence top, producing much lower optical depth and $T_{b}$ at $\mu-w$. Therefore, a much lower intensity of EUV lines is expected.

A check of this assumption could come only by performing $\mu-w$ and EUV observations of the same filament on the disk, which was the objective of this joint program of observations performed on July 28, 1996.

\section{Observations}

The filament site, located at $\simeq 45^{\circ} \mathrm{N}$ and $\simeq 30^{\circ}-45^{\circ} \mathrm{W}$, was observed by the SUMER, CDS and EIT instruments onboard SOHO and by the VLA at 2, 3.5, 6 and $21 \mathrm{~cm}$. Full disk observations at $1.7 \mathrm{~cm}$ (Nobeyama) were also available.

Due to space limitations, only one figure concerning some of the SUMER observations is presented here. More figures concerning observations withother instruments are shown by Alissandrakis et al. (1997). Below we will give a detailed description of the results obtained by all instruments involved in this campaign.

a) The filament appears as a dark feature with a shape very similar to the $H_{\alpha}$ filament at short radio wavelengths $(\lambda<21 \mathrm{~cm})$, in all images taken by CDS in the TR lines and by SUMER in the Ne VIII lines at $\lambda=770$ and $780 \AA$.

b) The filament does not appear at all in the images taken by SUMER in other TR lines at longer wavelengths such as those of Si IV at 1393 and $1402 \AA$ of O IV at 1401 and $1404 \AA$ and of C IV at 1548 and $1550 \AA$.

c) The filament appears as a dark feature, although with less defined contours than in the previously mentioned cases, at $\lambda=21 \mathrm{~cm}$ and in the coronal images observed by CDS and EIT.

In this paper we will not comment on these latter results, limiting our 


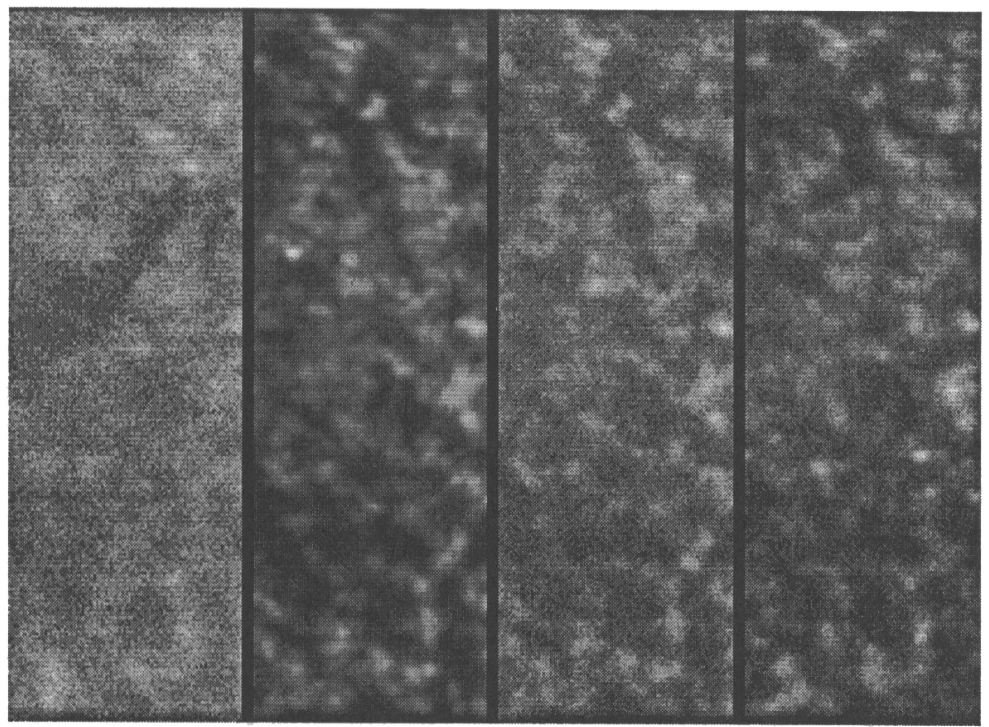

Figure 1. Monochromatic images of the filament region taken by SUMER. From left to right: Ne VIII at $770, \mathrm{Si}$ IV at $1393.8, \mathrm{O}$ IV 1401.2 and CIV at $1548 \AA$. Each field of view is $100 \times 296 \mathrm{arc}^{-\mathrm{sec}^{2}}$.

interpretation to the observations of Transition Region lines.

\section{Interpretation and Conclusions}

From the above observations it appears that the visibility of the filament in a given line formed in the Transition Region depends only on its wavelength, as shown by the O IV images: that at $\lambda=554 \AA$ (CDS), clearly shows the filament in absorption, while those at $\lambda=1401$ and $1404 \AA$ (SUMER) do not.

Although there are no observations in the range $780 \AA<\lambda<1393 \AA$, it appears reasonable to ascribe the observed absorption to the Lyman continuum and to assume that the wavelength separating the ranges where the filament is visible or not is $\lambda=912 \AA$.

The dark feature seen in all lines at $\lambda<912 \AA$ is therefore due to the Lycontinuum absorption of the radiation coming from the underlying Chromosphere-Corona Transition Region (CCTR), within the cool body of the optical prominence. (At shorter wavelengths the He I and He II continuum absorption could also contribute.) This absorption is clearly not balanced by the proper emission of PCTR above the filament. We may therefore conclude that the DEM in this latter case is much smaller than in the former. This is also confirmed by the much lower emission above the filament with respect to the surrounding background, seen at radio wavelengths, where the filament is totally thick and the observed flux comes only from the PCTR above the filament.

On the other hand, at all lines with $\lambda>912 \AA$, there is no absorption within the prominence, and the total intensity observed above the filament should be 
the sum of the CCTR emission and of the PCTR emission at its top. According to Schmahl and Orrall (1986) and Engvold et al. (1987), the EUV line intensities of the PCTR at the limb are $\sim 0.5$ and $\sim 0.3$ that of the quiet Sun. Assuming the same values above the filament, we would get an intensity ranging from 1.3 to 1.5 that of quiet Sun and the filament would clearly appear in emission. Since this is not the case, we must conclude that the EUV emission at the prominence top is smaller than that observed at its side.

Recent EUV observations made by Kucera et al. (1998, these proceedings) give a ratio of the PCTR to the CCTR line intensities at the limb equal to 0.1 , putting some doubt on the possibility of distinguishing the filament in emission on the disk. It must be pointed out that the Kucera et al. observation refers to an eruptive, not to a quiescent prominence which the present paper refers to.

Due to the large fluctuations among the few EUV line observations of prominences, we cannot affirm that this emission indicates a difference in the PCTR parameters at the different faces of the prominence, as it appears from the comparison with $\mu-w$ observations. It is clear that the only way to check it is by observing the same filament on the disk and at the limb at both wavelengths.

Acknowledgments.

The SUMER project is financially supported by DARA, CNES, NASA and ESA Prodex program (Swiss contribution). SOHO is a mission of international cooperation between ESA and NASA. One of the authors (FCD) wishes to acknowledge a grant of the Italian National Research Council (CNR), given to her as support for her stay at the Institut d'Astrophysique Spatiale. KB and JCV wish to thank the CNES for the financial support given to them for attending this meeting.

\section{References}

Alissandrakis, C.E., Drago, F., Bastian, T., Bocchialini, K., Delaboudinière, J.P., Lemaire, P., Vial, J.-C., Harrison, R.A, and Thompson, B. 1997, in Advances in the Physics of Sunspots, B. Schmieder, J.C. Toro del Iniesta and M. Vazquez (eds.), ASP Conf. Series, Vol. 118

Chiuderi, C. and Chiuderi Drago, F. 1990, Solar Phys., 132, 81

Chiuderi Drago, F. 1990, in Dynamics of Quiescent Prominences, V. Ruzdjak and E. Tandberg-Hanssen (eds.), Springer-Verlag, New York, p. 70

Engvold, O. 1989, in Dynamics and Structure of Quiescent Solar Prominences, E.R. Priest (ed.), Kluwer Acad. Publ., Dordecht, Holland, p. 47

Engvold, O., Kjeldseth-Moe, O. Bartoe, J-D.F. and Brueckner, G.E. 1987, 21st ESLAB Symp. ESA SP-275, p. 21

Leroy, J.L. 1989, in Dynamics and Structure of Quiescent Solar Prominences, E.R. Priest (ed.), Kluwer Acad. Publ., Dordrecht, Holland, p. 77

Schmahl, E.J. and Orrall, F.Q. 1986, in Coronal and Prominence Plasma, A. Poland (ed.), NASA Conf. Pub. 2442, p. 127 